\title{
Modeling And Pid Cascade Control For Uav Type Quadrotor
}

\author{
Renuka Ramkishan Choudhari ${ }^{1}$, Subhash.S. Sankeshwari ${ }^{2}$. \\ 1.Department Of Electrical Engineering, MBES College Of Engineering Ambejogai, India \\ 2. Department Of Electrical Engineering, MBES College Of Engineering Ambejogai, India
}

\begin{abstract}
This paper present the mathematical model of UAV type Quadrotor under the Newton-Euler formulation. A transfer function has been chosen which represents a brushless motor and its driver as one system. PID cascade control has been designed to solve the path tracking problem for quadrotor. The controller is evaluated in a $3 D$ environment in simulink.
\end{abstract}

Keyword: Cascade controller, PID controller, Quadrotor, UAV.

\section{Introduction}

Quadrotor is multirotor helicopter, which is also known as quadcopter. The most recent design of quadrotor is Unmanned Aerial Vehicle (UAV). A four rotor helicopter was firstly designed by Louis Breguet. In the year of 1920. Some of the groups have developed their own platform for UAV. Such as, Dragan- Flyer, the $\mathrm{X}$-UFO and the MD4-200. Quadrotor is useful tool for researchers to test and evaluate new ideas in number of different fields. Including flight control theory, navigation, real time system and robotics. UAV type quadrotor are also applicable for agriculture and film production. There are numerous advantages using quadrotor as a versatile test platforms. They are relatively cheap and available in variety of sizes, simple control mechanism, highly manuarable, and has potential to hover and take off, fly and land in small areas.

The most challenging part for the new generation, is to achieve greater performance. The various control techniques used for modeling and controlling of quadrotor such as LQR method, control of UAV using sliding mode control, Fuzzy controller, PID controller. In this paper we concentrate on stabilization problem of quadrotor in presence of actuator and sensor fault is considered. The dynamic describing the quadrotor parameters, which affect the dynamic of flying structure. The cascade control mechanism is used in this paper to achieve improved performance and reduce adverse effect. The main aim of this paper is to examine the effectiveness of designed attitude control with different type of PID controller.[1]. The paper is organized as follows, first the mathematical model of UAV type quadrotor is described. Second part presents the general structure of cascade control system and investigation of two type of PID controller with modified loop structure. This section include description of Type-A PID controller and Type-B PID controller.

\section{Quadrotor model}

The Structure of UAV type Quadrotor is simple one. Basically comprising of four rotors attached at the end of a symmetric cross. The main features that should be taken in such a structure are symmetry and rigidity. To avoid unstable flight structure should be as rigid as possible. The best way to achieve this is through use of lightweight alloys or composites.

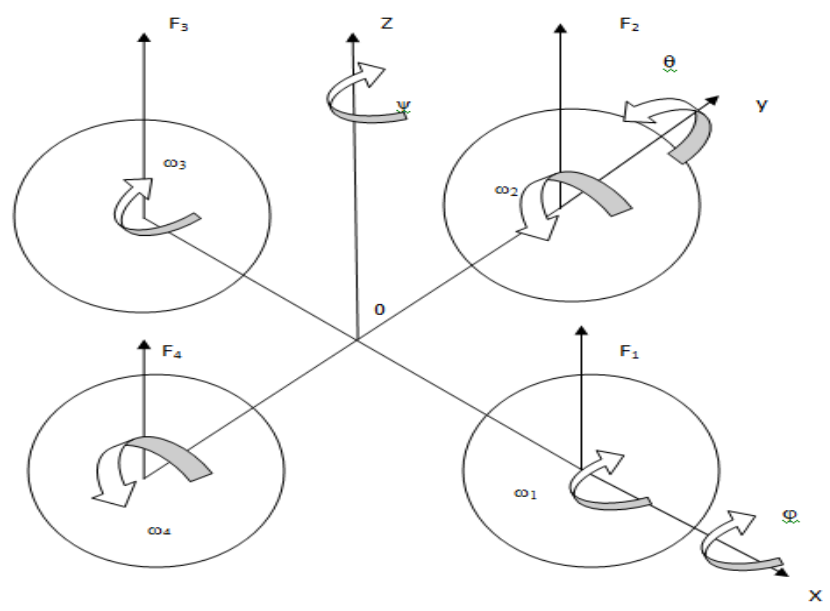

Figure 1. Quadrotor Thrust aerial vehicle 
The two pair of propeller as shown in figure1, $(1,3)$ and $(2,4)$ rotates in apposite direction .The pair $(1,3)$ rotates clockwise and remaining pair $(2,4)$ rotates anticlockwise. This combination of rotation produce apposite torque. This results propellers generate vertical lifting force upward which raises quadrotor body in the air and it can moves in pitch, roll, yaw, hover, take off and landing. Pitch and roll movement can be achieved by altering the speed of any one pair of motor. while other motor pair speed remain constant. Yaw movement can be achieved by altering the speed of both motor pairs in quadrotor.

\section{Mathematical model}

The UAV type quadrotor is six degree of freedom system.

$$
\begin{gathered}
\mathrm{X}=[\varphi, \theta, \psi, \mathrm{x}, \mathrm{y}, \mathrm{z}, \dot{\varphi}, \dot{\theta}, \dot{\psi}, \dot{x}, \dot{y}, \dot{z}] \\
\mathrm{U}=\left[\mathrm{u}_{1}, \mathrm{u}_{2}, \mathrm{u}_{3}, \mathrm{u}_{4}\right] . \text { where } \mathrm{u}_{\mathrm{i}}-\text { control input } \\
\mathrm{i}=1,2,3,4-\text { motor input. } \\
\mathrm{u}_{1}=\mathrm{b}\left(\omega_{1}{ }^{2}+\omega_{2}{ }^{2}+\omega_{3}{ }^{2}+\omega_{4}{ }^{2}\right) \\
\mathrm{u}_{2}=\mathrm{b}\left(-\omega_{2}{ }^{2}+\omega_{4}{ }^{2}\right) \\
\mathrm{u}_{3}=\mathrm{b}\left(\omega_{1}{ }^{2}-\omega_{3}{ }^{2}\right) \\
\mathrm{u}_{4}=\mathrm{d}\left(-\omega_{1}{ }^{2}+\omega_{2}{ }^{2}-\omega_{3}{ }^{2}+\omega_{4}{ }^{2}\right)
\end{gathered}
$$

The relation between the kinetic energy and potential energy is represented by Lagrange equation

$$
\begin{aligned}
& \mathrm{L}=\mathrm{D}_{\mathrm{k}}-\mathrm{P} \\
& \mathrm{F}=\frac{d}{d t}\left(\frac{\partial L}{\partial \dot{X}}\right)-\frac{\partial L}{\partial X}
\end{aligned}
$$

where; $\mathrm{L}$ is Lagrangian, $\mathrm{D}_{\mathrm{k}}$ is Kinetic energy, $\mathrm{P}$ is potential energy, $\mathrm{X}=[\mathrm{x}, \mathrm{y}, \mathrm{z}, \varphi, \theta, \psi]^{\mathrm{T}}$ is a vector of generalized coordinates, $\quad \mathrm{F}=\left(\mathrm{F}_{\mathrm{E}}, \mathrm{M}\right)$ are generalized forces coordinates and moments $\mathrm{M}$ applied to the quadrotor due to control input. For translation motion the Lagrange equation has the form:

$$
\mathrm{F}_{\mathrm{E}}=\frac{d}{d t}\left(\frac{\partial L}{\partial \dot{\varepsilon}}\right)-\frac{\partial L}{\partial \varepsilon}
$$

where: $\Sigma=[\mathrm{x}, \mathrm{y}, \mathrm{z}]^{\mathrm{T}}-$ Position coordinates

$$
\begin{aligned}
& \mathrm{F}_{\mathrm{E}}=\left[\begin{array}{c}
\sin \theta \\
-\sin \varphi \cos \theta \\
\cos \varphi \cos \theta
\end{array}\right] . \mathrm{f}_{\mathrm{g}} \\
& \mathrm{f}_{\mathrm{g}}=\mathrm{F}_{1}+\mathrm{F}_{2}+\mathrm{F}_{3}+\mathrm{F}_{4} \\
& \mathrm{~F}_{\mathrm{i}}=\mathrm{b} \omega_{\mathrm{i}}{ }^{2}
\end{aligned}
$$

$\omega_{i}$ - Rotor speed, b - Thrust factor

The Lagrange equation for rotational motion is represented as follows:

$$
\mathrm{T}=\frac{d}{d t}\left(\frac{\partial L}{\partial \dot{\eta}}\right)-\frac{\partial L}{\partial \eta}
$$

Where: $\eta=[\varphi, \theta, \psi]^{\mathrm{T}}$ - Euler angles

$$
\begin{aligned}
& \mathrm{T}=\left[\mathrm{T}_{\varphi} . \mathrm{T}_{\theta}, \mathrm{T}_{\psi}\right]^{\mathrm{T}} \\
& \mathrm{T}_{\varphi}=\mathrm{bl}\left(\omega_{4}{ }^{2}-\omega_{2}{ }^{2}\right)-\mathrm{I}_{\mathrm{r}} \dot{\theta}\left(\omega_{1}+\omega_{3}+\omega_{2}-\omega_{4}\right) \\
& \mathrm{T}_{\theta}=\mathrm{bl}\left(\omega_{3}{ }^{2}-\omega_{1}{ }^{2}\right)+\mathrm{I}_{\mathrm{r}} \dot{\varphi}\left(\omega_{1}+\omega_{3}+\omega_{2}-\omega_{4}\right) \\
& \mathrm{T}_{\psi}=\mathrm{d}\left(\omega_{1}{ }^{2}-\omega_{2}{ }^{2}+\omega_{3}{ }^{2}-\omega_{4}{ }^{2}\right)
\end{aligned}
$$

Above equation $T_{\varphi} T_{\theta} T_{\psi}$ consist of action of thrust forces difference of each pair. The quadrotor dynamic model

with $\mathrm{X}, \mathrm{Y}, \mathrm{Z}$ motion as consequence of pitch, roll and yaw rotation is as follows.

$$
\ddot{\theta}=\frac{1}{\mathrm{Ixx}}\left(-\dot{\varphi}^{2}\left(\mathrm{I}_{\mathrm{xx}}-\mathrm{I}_{\mathrm{zz}}\right) \mathrm{s}(\theta) \mathrm{c}(\theta)-\dot{\varphi} \dot{\psi} \mathrm{I}_{\mathrm{zz}} \mathrm{c}(\theta)+\mathrm{T}_{\theta}\right)
$$

$$
\varphi=\frac{1}{\operatorname{Iyy}(1+\mathrm{s} 2(\theta))}\left(-\ddot{\psi} \mathrm{I}_{\mathrm{zz}} \quad \mathrm{s}(\theta \quad)-\dot{\theta} \dot{\varphi} c(\theta)\right.
$$

(8)

$$
\begin{aligned}
& \left.\left(2 \mathrm{I}_{\mathrm{zz}}-2 \mathrm{I}_{\mathrm{yy}}\right)-\dot{\theta} \dot{\psi} \mathrm{c}(\theta)+\mathrm{T}_{\varphi}\right) \\
& \ddot{\psi}=\frac{1}{\mathrm{Izz}}\left(-\ddot{\varphi} \mathrm{I}_{\mathrm{zz}} \mathrm{s}(\theta)+\mathrm{T}_{\psi}\right)
\end{aligned}
$$

$$
\begin{gathered}
\ddot{y} \cdot \mathrm{m}=-\mathrm{f}_{\mathrm{g}} \mathrm{c}(\theta) \mathrm{s}(\varphi) \\
\ddot{z} \cdot \mathrm{m}+\mathrm{g}=\mathrm{f}_{\mathrm{g}} \mathrm{c}(\theta) \mathrm{c}(\varphi)
\end{gathered}
$$

where: $c$ and s are abbreviations of 'sin' and 'cos', $\mathrm{I}_{\mathrm{xx}}, \mathrm{I}_{\mathrm{yy}}, \mathrm{I}_{\mathrm{zz}}$ are inertia moments. 


\section{Control Scheme}

PID controller has been applied for attitude control of UAV type quadrotor. The PID controller is applied to regulate both position and orientation (angular momentum) of quadrotor. The performance of PID controller indicate good attitude stabilization. The time response is good, with almost zero steady state error in order to achieve better performance and reduce external disturbance. The cascade controller consist of two loops inner loop and outer loop.

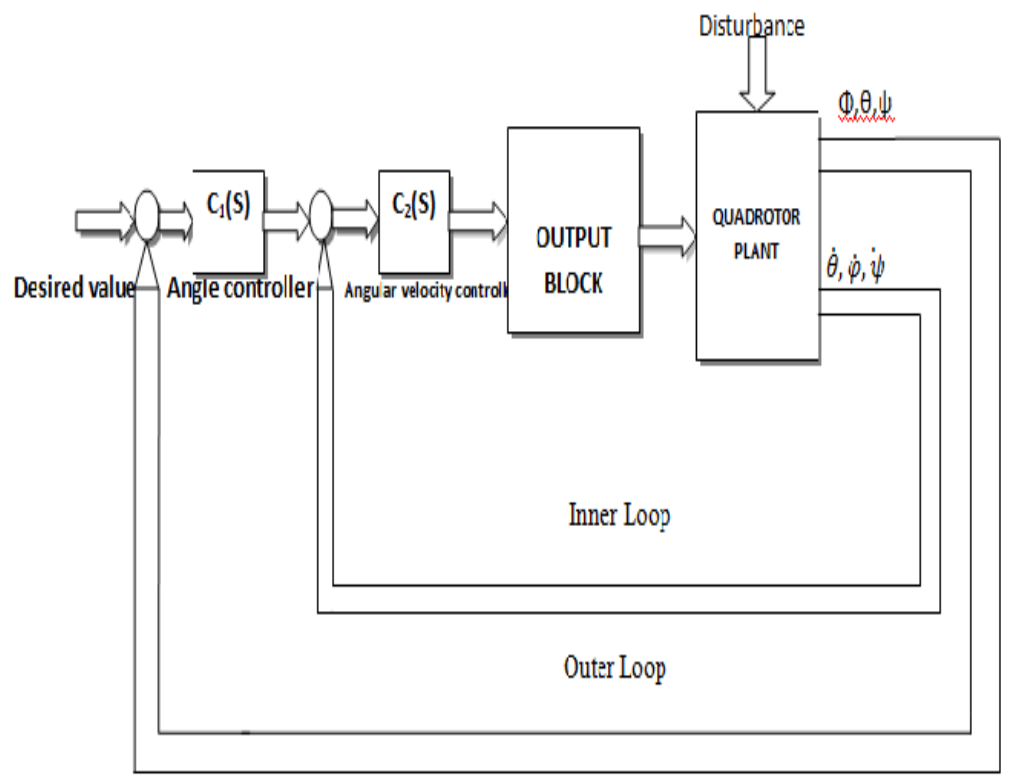

Figure 2: Cascade controller

The geometry of this block diagram as shown in figure 2 defines inner loop involving secondary controller and outer loop involving primary controller. The inner loop function like a traditional feedback control with set point, a system variable and controller acting on system by means of actuator. In this paper cascade controller perform control task as an angular stabilization. The angular velocity of rotating platforms are additional measurement that can be used in inner loop. The outer loop is based on Euler angles. The block diagram of cascade control for UAV type quadrotor is as shown in figure2. In both loop three type of PID controllers are considered.

\section{1) Type-A PID Controller}

In control theory PID controller is represented in continues time domain is

$$
\mathrm{u}(\mathrm{t})=\mathrm{k}_{\mathrm{p}} \mathrm{e}(\mathrm{t})+\mathrm{k}_{\mathrm{i}} \int_{0}^{t} e(t) d t+\mathrm{k}_{\mathrm{d}} \frac{d e(t)}{d t}
$$

where; $k_{p}$ is proportional gain, $k_{i}$ is integral gain and $k_{d}$ is derivative gain. Type-A PID controller consist of proportional gain, integral gain, derivative gain. Block diagram of PID controller is shown in figure 3

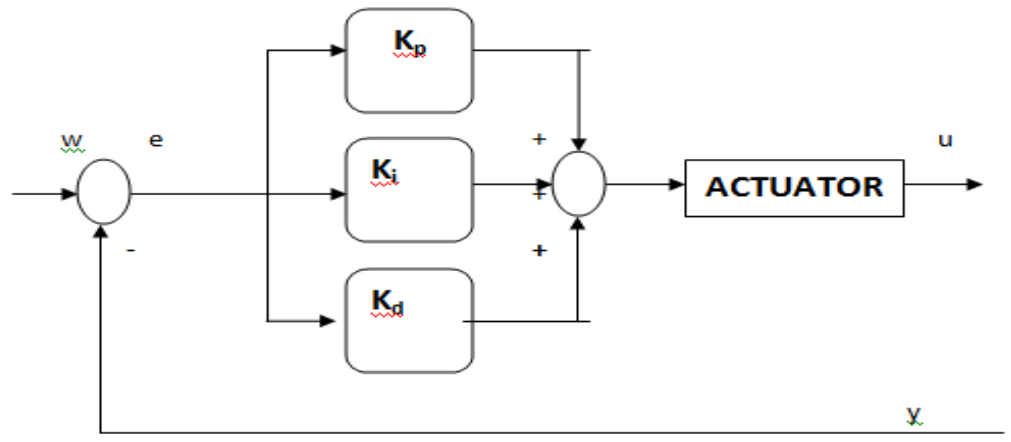

Figure 3: Type-A PID Controller 


\section{2) Type-B PID Controller}

The process of setting the optimal gain to get ideal response from control system is known as tuning of PID controller. In most practical control system very small derivative term is used, because derivative response is highly sensitive to noise in variables. Therefore in type-B PID controller, we minimize the derivative gain. Figure 4 represents the block diagram of Type-B PID controller.

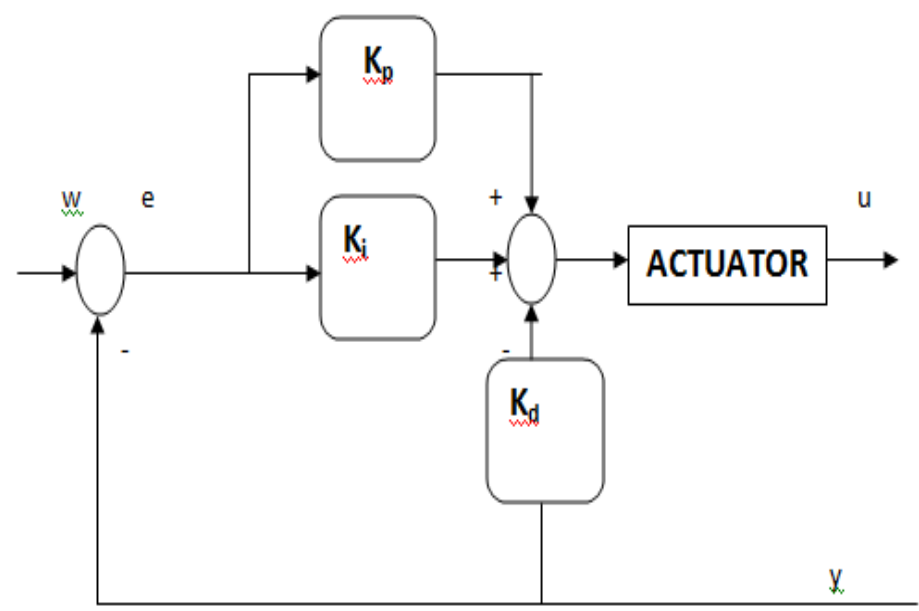

Figure4:Type-B PID controller

The mathematical equation for Type-B PID controller is;

$$
\mathrm{u}(\mathrm{t})=\mathrm{k}_{\mathrm{p}} \mathrm{e}(\mathrm{t})+\mathrm{k}_{\mathrm{i}} \int_{0}^{t} e(t) d t-\mathrm{k}_{\mathrm{d}} \frac{d y(t)}{d t}
$$

In general increasing $\mathrm{k}_{\mathrm{p}}$, speed of control system increases. The integral term response will be continually increasing unless error is zero. Therefore the PID controller with minimum value of $\mathrm{k}_{\mathrm{d}}$ gives good results, as compare to Type-A PID controller.

\section{3) Type-C PID controller}

Mathematical equation for Type-C PID controller is as follows:

$$
\mathrm{u}(\mathrm{t})=-\mathrm{k}_{\mathrm{p}} \mathrm{y}(\mathrm{t})+\mathrm{k}_{\mathrm{i}} \int_{0}^{t} e(\mathrm{t}) \mathrm{dt}-\mathrm{k}_{\mathrm{d}} \frac{d y(t)}{d t}
$$

Block diagram for Type C PID controller is

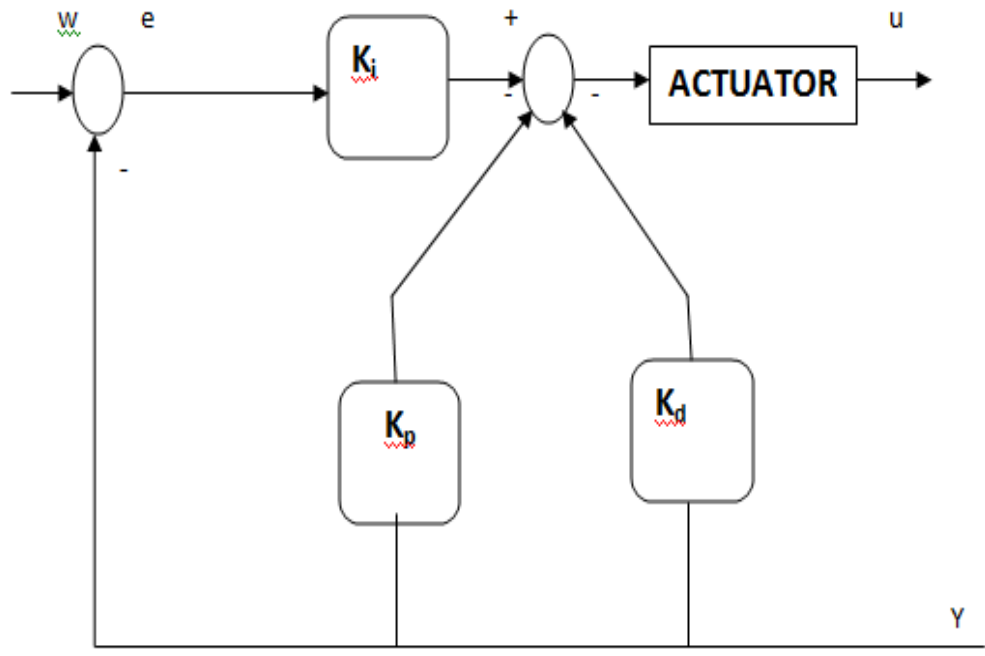

Figure 5 : Type C PID Controller 


\section{Simulation Results}

In this section we present the simulation results which are conducted to evaluate performance of designed attitude control system in cascade structure with different type of PID controller. In design process we consider two type of PID controller optimizing the parameters in view of the assumed reference model. Feedback data for regulators are six variables: Euler angles $\theta, \psi, \varphi$ (outer loop), angular velocities $\dot{\theta}, \dot{\psi}, \dot{\varphi}$ (inner loop). Control signals are motor input $\mathrm{u}_{1}, \mathrm{u}_{2}, \mathrm{u}_{3}, \mathrm{u}_{4}$.

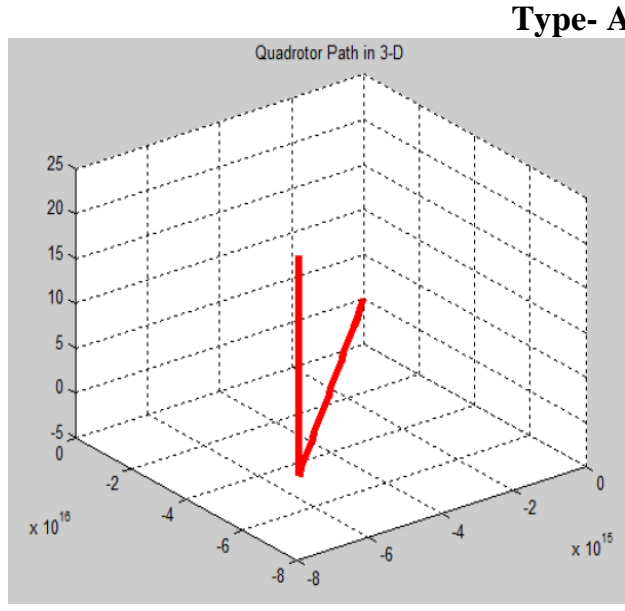

Figure 6: Quadrotor 3D path

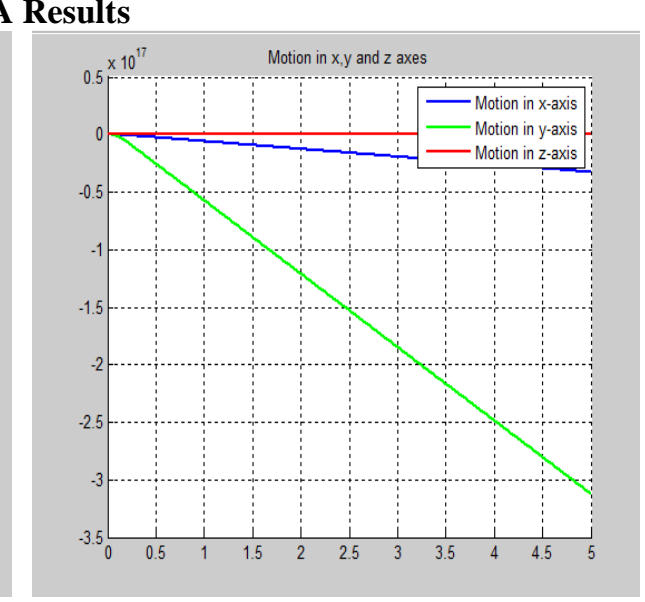

Figure 7: Motion in XYZ plane

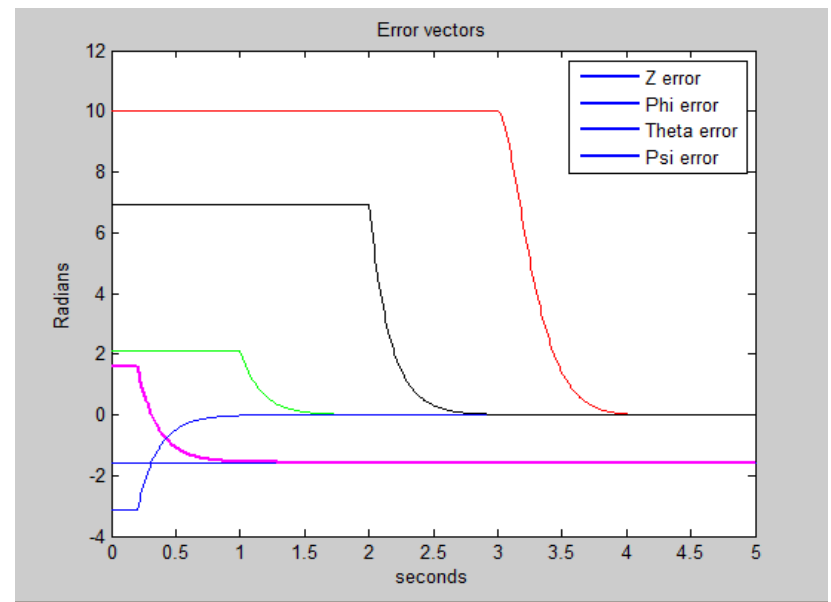

Figure 8: Error response

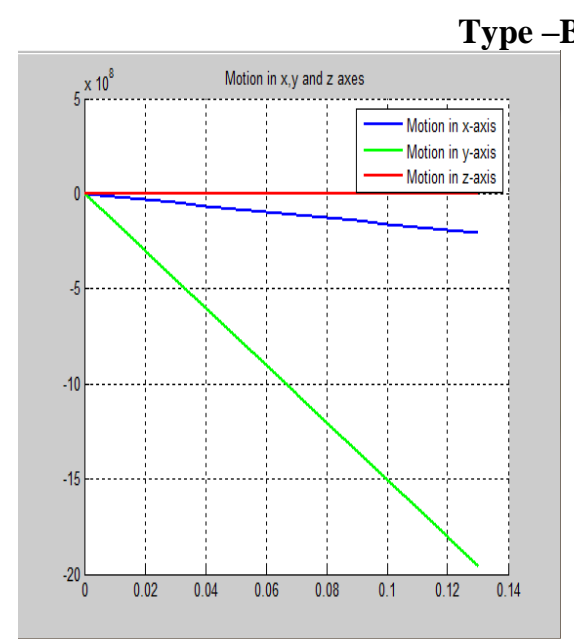

Figure 9: Motion in xyz plane

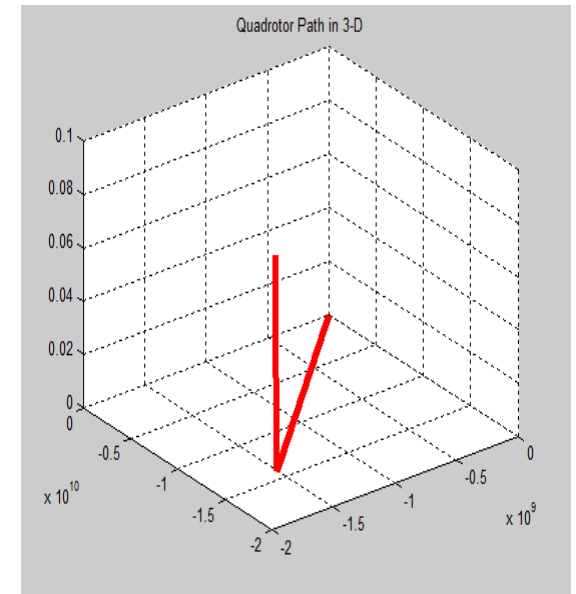

Figure 10: Quadrotor 3D path 


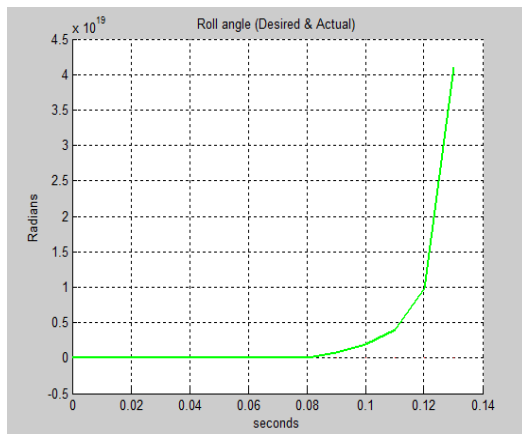

Figure 11: Roll angle Response

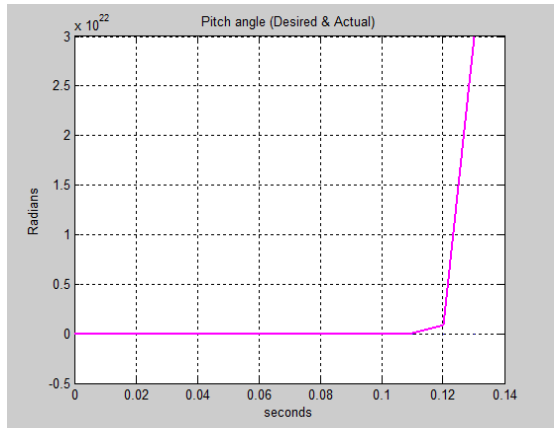

Figure 12: Pitch angle Response

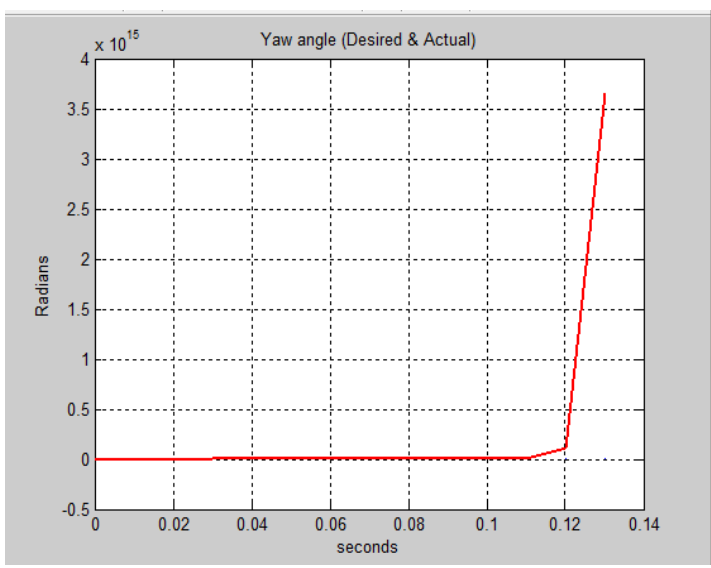

Figure 13: Yaw angle response

Type-C results

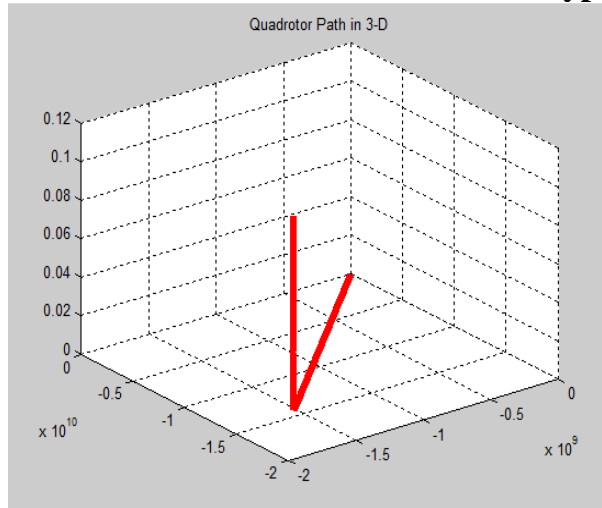

Figure14 Quadrotor 3D path

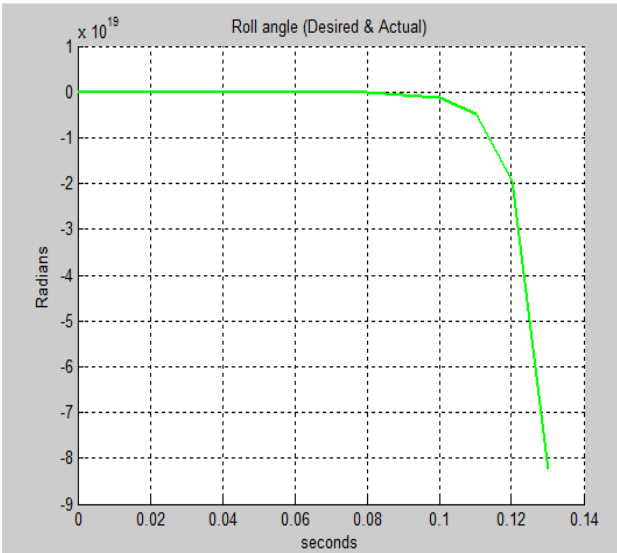

Figure 16: Roll angle response

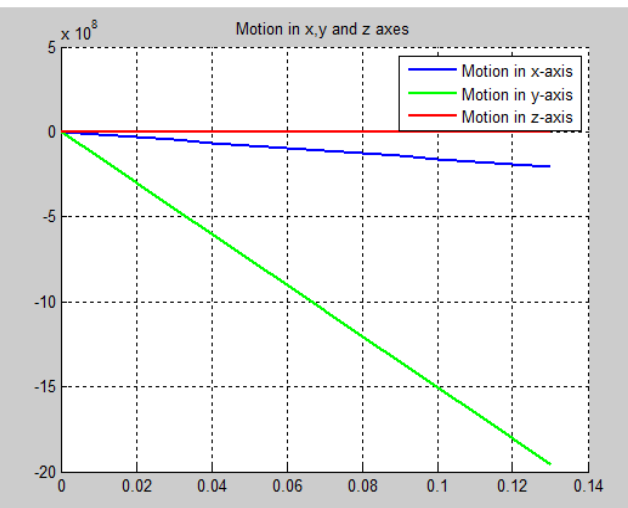

Figure 15: Motion in xyz plane

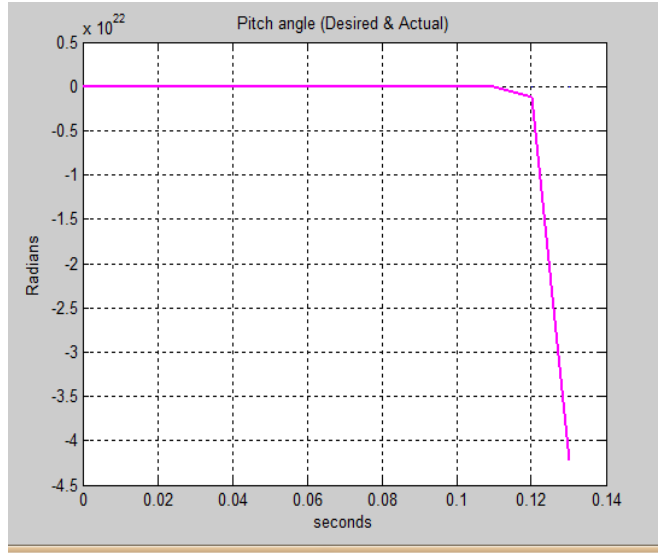

Figure 17: Pitch angle response 


\section{Conclusion}

In this paper the three approaches of PID controller are considered to the problem of attitude control of UAV type Quadrotor. Main goal of this research is to achieve the angular response for different approaches of PID controller. Three architecture are presented and examined with best performance. All the reviewed architecture of controller resulted in almost same output response. The application of cascade control structure gives the possibility to adapts the simple PID algorithm for controlling complex system, such as vertical take-off and landing platforms. As PID controller gives steady state error zero, so the system becomes more stable. Angular stabilization is obtained by using PID controller for UAV type quadrotor

\section{References}

[1]. H.L. Wade, "Basic and advanced regulatory control system design and application," ISA, United state of America, 2004

[2]. S. Bouabdallah, A Noth, and R. Siegwart, " PID vs LQ Control Techniques applied to an indoor micro Quadrotor", Proc. Of Int. Conf. On intelligent Robot and Systems, Japan, 2004.

[3]. A. Tayebi and S.McGilvray, "attitude stabilization of a VOLT quadrotor aircraft", IEEE trans. on control system technology, vol. 14 , no. 3, 2006, pp. 562-571

[4]. K.P Valavanis, advances in Unmanned Aerial Vehicles. The Netherland: Springer-Verlag, 2007.

[5]. P. Lindahl, E. Moog and S.R. Shaw, Simulation design and validation of UAV propulsion system. 2009.

[6]. R. Goel, S. M. Shah, N.K. Gupta, N. Ananthkrishnan, 2009. Modelling, Simulation and flight of an Autonomous Quadrotor. Proceeding of ICEAE.

[7]. Hongning Hou. Jian Zhuag, Hu Xia,Guanwei wang and Dehong Yu."A simple control of UAV type Quadrotor" in Mechatronics and automation (ICMA), 2010.

[8]. Farid Kendoul. Survey of advance in guidance, navigation and control of unmanned rotorcraftsystem. Journal field robotics 29 (2), 2012 